\title{
A proximal femoral implant preserves physiological bone deformation: a biomechanical investigation in cadaveric bones
}

\author{
W Macdonald ${ }^{1 *}$, L V Carlsson ${ }^{1,2}$, C M Jacobsson ${ }^{1}$ and T Q Lee ${ }^{3}$ \\ ${ }^{1}$ Department of Biomaterials/Handicap Research, Institute for Surgical Sciences, University of Gothenburg, \\ Gothenburg, Sweden \\ ${ }^{2}$ Department of Orthopaedics, Sahlgrens University Hospital, University of Gothenburg, Gothenburg, Sweden \\ ${ }^{3}$ Orthopaedic Biomechanics Laboratory, VA Long Beach Healthcare System, Long Beach, California, USA
}

\begin{abstract}
The aim of this study was to compare the perturbances in bone deformation patterns of the proximal femur due to a conventional cemented femoral stem and a novel uncemented implant designed on the principles of osseointegration. Five matched pairs of fresh frozen human femora were mechanically tested. Bone deformation patterns, measured with a video digitizing system under $1.5 \mathrm{kN}$ joint force, showed that the cemented Spectron femoral implant caused significant alterations to the proximal femoral deformation pattern, whereas the Gothenburg osseointegrated titanium femoral implant did not significantly alter the bone behaviour $(p<0.05)$. Vertical micromotions measured under $1 \mathrm{kN}$ after 1000 cycles were within the threshold of movement tolerable for bone ingrowth $(21 \mu \mathrm{m}$ for the Gothenburg system and $26 \mu \mathrm{m}$ for the cemented implant).
\end{abstract}

Keywords: bone implant system, non-cemented hip, fresh human hips, implant stability, load transfer

\section{NOTATION}

$\begin{array}{ll}\text { A-P } & \begin{array}{l}\text { antero-posterior } \\ \text { M-L }\end{array} \\ Q \text { angle } & \begin{array}{l}\text { medio-lateral } \\ \text { angle between the femoral shaft and the } \\ \text { mechanical axis (centre of the femoral } \\ \text { head to the mid-line of the femoral } \\ \text { condyles at the knee) } \\ \text { video digitizing system }\end{array}\end{array}$

\section{INTRODUCTION}

In the search for improved endurance of total hip arthroplasty components, two concerns have been uppermost. Failure of acrylic cement and peri-implant osteolysis have encouraged many researchers to attempt cementless fixation, despite the secure fixation provided by acrylic bone cement for the majority of patients. After initially encouraging results, many studies found excessive

The MS was received 14 January 2002 and was accepted after revision for publication on 2 August 2002.

* Corresponding author: 5 St James Square, Boscombe, Dorset BH5 $2 B X, U K$. proximal bone loss through resorption, which in turn destabilized the femoral component $[\mathbf{1}, \mathbf{2}]$.

Generalized osteolysis might be attributable to 'cement disease' [3, 4] or 'particle disease' [5], but proximal loss must also implicate mechanically induced resorption due to stress shielding [6-10]. Proximal stress shielding has been shown to be related to stem design and material $[\mathbf{1 1}, \mathbf{1 2}]$, and designs have been altered to increase proximal stress transfer $[\mathbf{1 3}, \mathbf{1 4}]$ with variable success $[15,16]$.

Predictable stress transfer to bone has been shown to be achievable under the principles of osseointegration; first developed in oral and maxillofacial implants [17-19]. Preliminary investigations by the current authors predicted that the principles could be transferred to the orthopaedic situation [20-22], and design calculations confirmed that optimal proximal stress transfer could be achieved at the hip with such designs. Practical demonstration of the improved load transfer to the human anatomy was required.

Proximal loading has been investigated by measuring bone surface strains $[\mathbf{2 3}, \mathbf{2 4}$ ], bone deformations [25] and implant-bone micromotion [26, 27]. Bone deformation measurement has been shown to be quick, reproducible and minimally invasive [25], and was selected for the investigation of the mechanical effects of an 
osseointegrated femoral component. Due to its excellent clinical results in the Swedish National Hip Registry [28], the Spectron cemented femoral implant was selected as a control system for comparison.

\section{MATERIALS AND METHODS}

The experimental procedure was generally similar to that already used for the comparison of two press-fit cementless femoral stems [25]. After radiographing the intact femora, their proximal deformation pattern was determined by a video digitizing system (VDS) during mechanical testing. Proximal femoral replacement was then performed with a cemented or cementless system, the proximal deformation patterns were again characterized by VDS and bone-implant micromotion was measured.

\subsection{Femoral preparation}

Five matched pairs of fresh frozen human femora were obtained from autopsy, stripped of all soft tissues and the entire femoral length was measured with calipers. Antero-posterior (A-P) and lateral radiographs were obtained at 120 per cent magnification. The femora were then potted with the distal condyles fixed by eight fixation pins embedded in dental stone inside a steel tube and with the shaft vertical $\left(0^{\circ}\right.$ flexion and $0^{\circ}$ adduction). Small circular reflective markers $(5 \mathrm{~mm}$ in diameter) were placed on each femur at anatomically reproducible locations, in both anterior and medial views (Fig. 1).

The centre of the femoral head was determined by its projection in the three orthogonal planes, and this was marked in the A-P and medio-lateral (M-L) planes (point 1; Fig. 2). From the previously obtained femoral length, exactly half the length was marked on the projected shaft centre-lines in both views (point 5; Fig. 2). Four more points, 6 and $12 \mathrm{~cm}$ proximal and distal to this point, were then marked on the centre-lines (points 3 and 4 , and 6 and 7).

The centre-line of the femoral neck was traced to its intersection with the femoral shaft centre-line in both planes, and these two intersection points were marked (point 2). The angle between the two lines in the frontal plane represents the true femoral neck angle, and that in the transverse plane the femoral neck anteversion. Finally a marker was placed distally to locate the centre of the knee and so define the mechanical axis; this can be approximated by a line between the centre of the femoral head and the centre of the knee.

For the mechanical testing, the femora were positioned in the materials testing machine (Model 5500R, Instron Corp. Canton, Massachusetts) in the anatomical position, with the mechanical axis vertical and the femoral shaft axis adducted accordingly. At the knee, the $Q$ angle

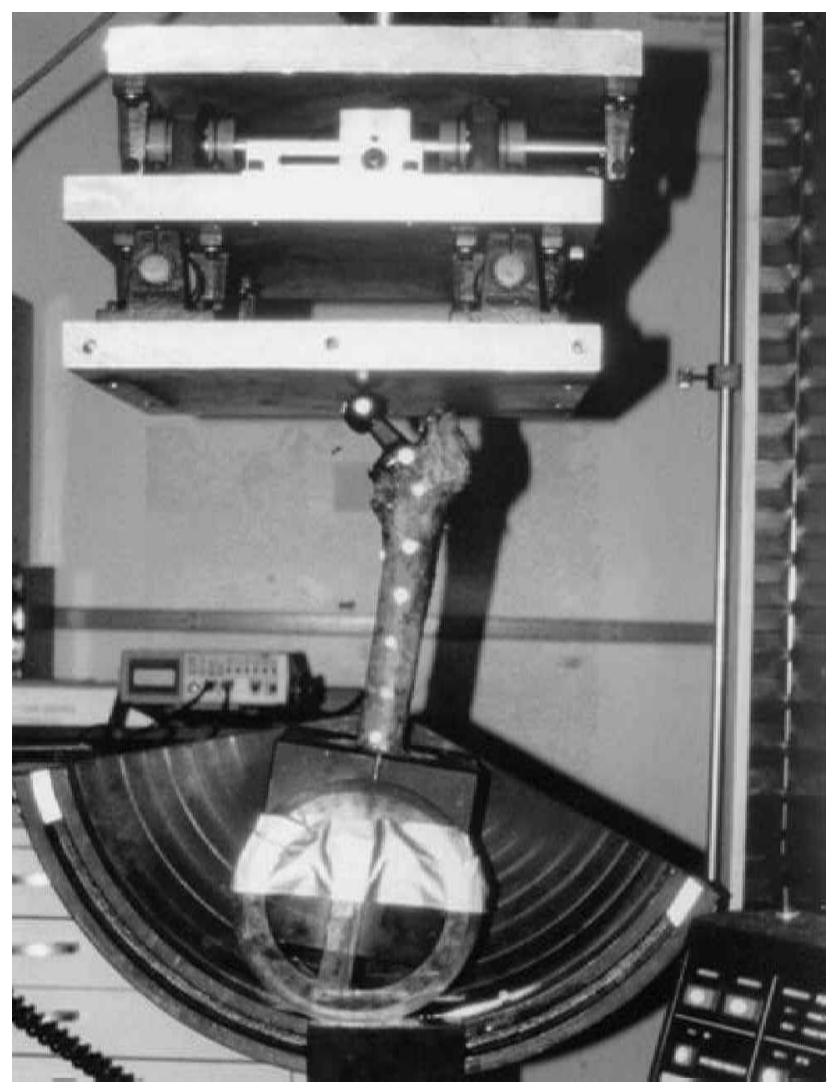

Fig. 1 Implanted femur positioned in test machine for deformation measurement; antero-posterior view (Spectron implant)

is defined as the angle between the line of action of the quadriceps and the patellar tendon. In an isolated femur this approximates to the angle between the femoral shaft and the mechanical axis. Testing orientations were then defined at $Q+0, Q+10^{\circ}$ and $Q+20^{\circ}$.

After measurement of anteversion and neck length, and testing of the proximal femoral deformation pattern of the intact bones (as described below), one femur of each pair was implanted with a cemented Spectron and the contralateral femur with a cementless Gothenburg arthroplasty. The left-right allocation of the two designs was randomized.

Templating of the radiographs indicated the probable size and orientation of the implant, and once the femur had been surgically prepared using the manufacturer's instrumentation, this implant selection was confirmed or corrected and the appropriately sized implant was positioned in situ. All arthroplasties were undertaken by one orthopaedic surgeon with extensive experience in both techniques.

The Spectron implants were inserted with thirdgeneration cementing techniques, using Palacos $\mathrm{R}$ cement (with gentamicin) of regular viscosity. The cementing technique included brushing and pressure lavage of the intramedullary canal, tamponade to dry the cavity, vacuum cement mixing and pressurized delivery by injection gun 

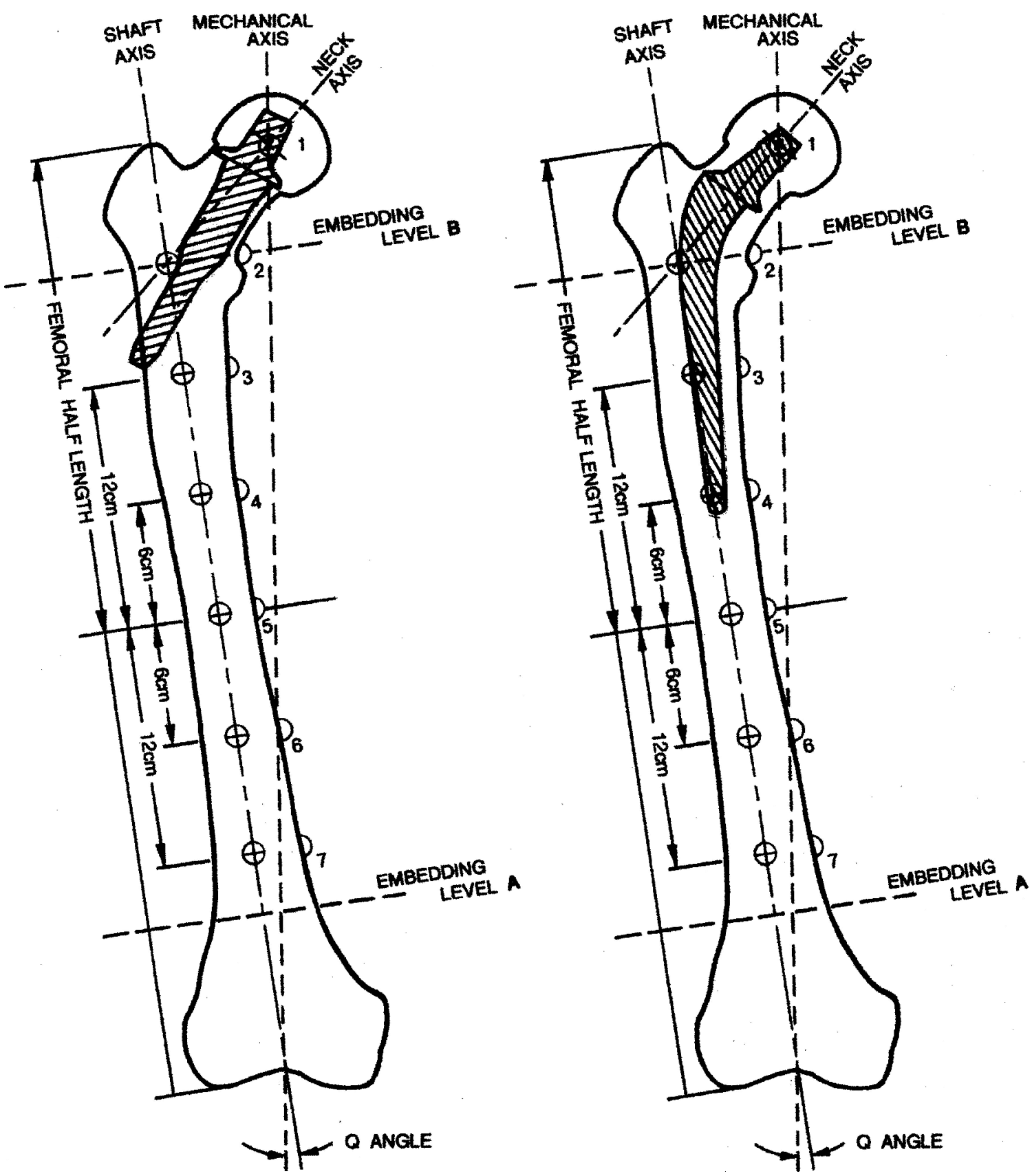

Fig. 2 Schematic diagram of femoral marking scheme, axes and angles (showing approximate implanted position of Gothenburg and Spectron components)

with cement restrictors proximally and distally. The implant was inserted late into 'thickening' cement. At least a full hour was allowed for curing of the cement before mechanical testing was undertaken.

Cementless Gothenburg arthroplasty was performed using the dedicated instrumentation with accurate preparation of the bony implant bed [29], and the definitive implant was inserted. The construct as tested thus represents the immediate postoperative stability of the cementless device, ignoring any improvement to the interfacial properties which might occur due to osseointegration. Similarly, because longer curing of the cement will increase the strength and modulus of the cemented construct, the latter performed in the testing 'less stiffly' than the final result in clinical practice. The implant-bone constructs were then subjected to proximal deformation pattern and micromotion investigations, using VDS, as described below.

\subsection{Mechanical testing}

The accuracy and precision of the VDS have previously been established to be better than 1 per cent and $1 \mu \mathrm{m}$ 
respectively [30]. Once the femoral construct had been marked for VDS measures, each femur was positioned in turn in the Instron machine at orientations of $Q+0^{\circ}$, $Q+10^{\circ}$ and $Q+20^{\circ}$ for non-destructive cyclic loading to determine proximal deformation patterns (Fig. 1). Two additional markers were added on the anterior surface of the intertrochanteric region of the femur to improve the characterization of the deformations in this region. Twelve cycles of vertical compressive load, from 150 to $1500 \mathrm{~N}$, were then applied at a cross-head speed of $100 \mathrm{~mm} / \mathrm{min}$ through a linear bearing. A pair of linear bearings, oriented perpendicular to the direction of loading, allowed free translation of the femoral head in the transverse plane in response to the loading action. Tests were undertaken separately with A-P video imaging and with M-L imaging.

Previous tests had established [25] that an appropriate level for testing was $1500 \mathrm{~N}$-about two-thirds of the ultimate strength. The loading speed of $100 \mathrm{~mm} / \mathrm{min}$ was dictated by the optimum sampling speed of the VDS data collection system. After proximal femoral deformation had been measured for the implanted femora (Figs 3 and 4), the specimens were repotted for implantbone micromotion measurements. For these measures the femur was supported and embedded within a tube reaching proximally to the femoral neck-shaft junction (embedding level B, Fig. 2). Two additional reflective markers of $1 \mathrm{~mm}$ diameter were applied, one placed on the medial edge of the implant collar and one on the adjacent bone.

For implant micromotion tests, each hip was subjected to 1005 cycles of axial compressive load cycled from 100 to $1000 \mathrm{~N}$ at a cross-head speed of $100 \mathrm{~mm} / \mathrm{min}$ and a simultaneous internal torque of $10 \mathrm{~N} \mathrm{~m}$. These loads correspond to those reported for maximal single limb stance and torsional moments measured by telemetered femoral components [31]. The relative micromotion between the two markers in the vertical direction was measured with the VDS at intervals of 100 cycles, commencing at the fifth cycle. Rotational micromotion was calculated as the absolute value of the change in horizontal distance between the two markers.

\subsection{Analysis}

The VDS system defines the cyclic motion of each marker point in response to the loading cycle. The marker at mid-shaft is set as the origin, so that motions at each marker had to be corrected for motion of the jig or testing machine and adjusted back to the reference point of the distal bone centre. Measurements were made intact and after prosthesis implantation, and results were then paired for intact and operated femora by loading condition and by marker level. Matched-pair analysis of variance was used to compare movements pre- and postimplantation and between the two prosthesis types. Post
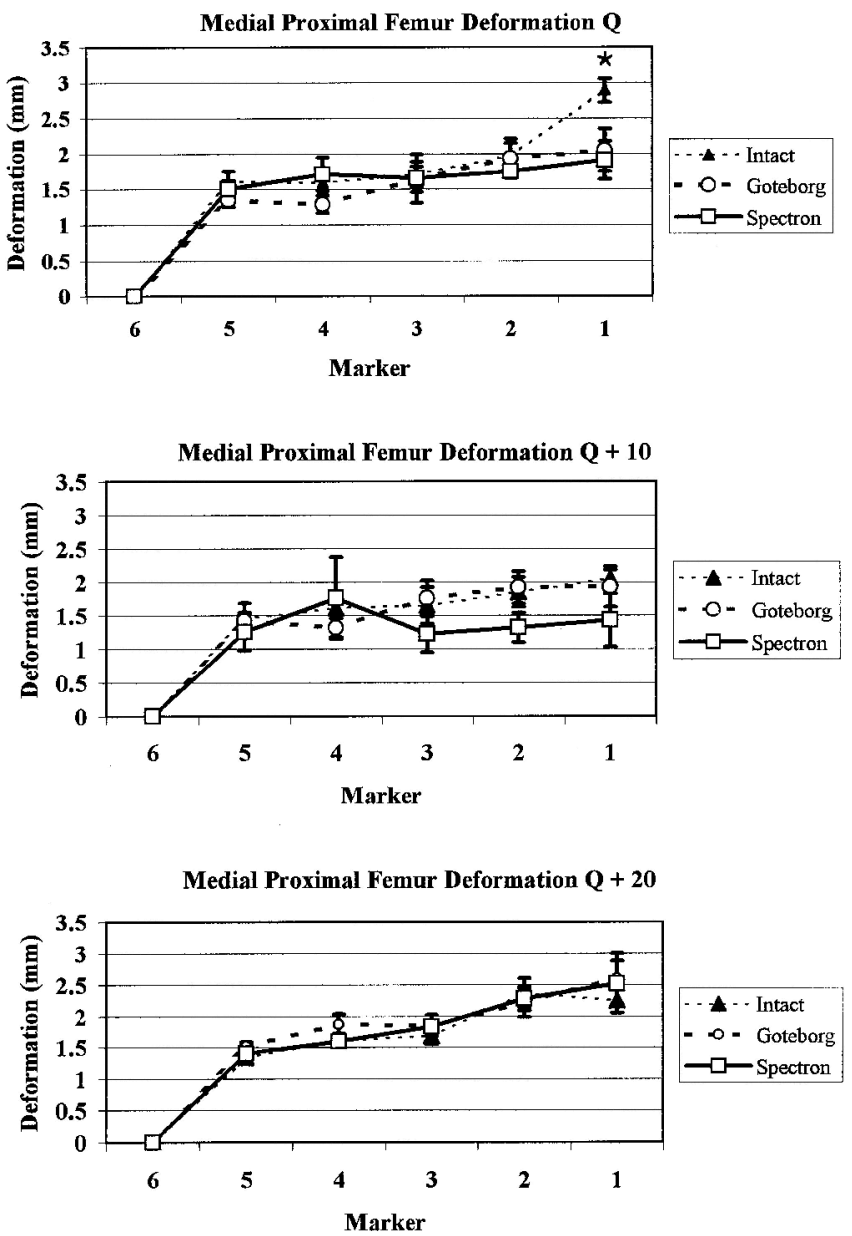

Fig. 3 Medio-lateral measures of proximal femoral deformation patterns (means \pm one standard error). * Statistically significant difference between the intact femur and the Spectron and between the intact femur and the Gothenburg

hoc comparisons including Scheffe's test were used to identify individual group mean differences.

\section{RESULTS}

Mean femoral neck angle and anteversion for the five femora used for the Spectron implant were $136 \pm 2^{\circ}$ and $9 \pm 2^{\circ}$ before implantation, and those for the Gothenburg implant were $133 \pm 2^{\circ}$ and $10 \pm 1^{\circ}$. After arthroplasty, the Spectron system had achieved $146 \pm 4^{\circ}$ and $12 \pm 7^{\circ}$, while the Gothenburg system had achieved $149 \pm 2^{\circ}$ and $12 \pm 2^{\circ}$. Matched-pair comparisons between the intact bone and its implanted construct were statistically significant $(p<0.01)$ for the Spectron system and for the Gothenburg implant individually $(p<0.05)$, but there was no statistically significant difference between the implanted reconstructions of the two systems $(p>0.5)$.

Whole femoral stiffness overall, before and after implantation, was significantly increased for both the 

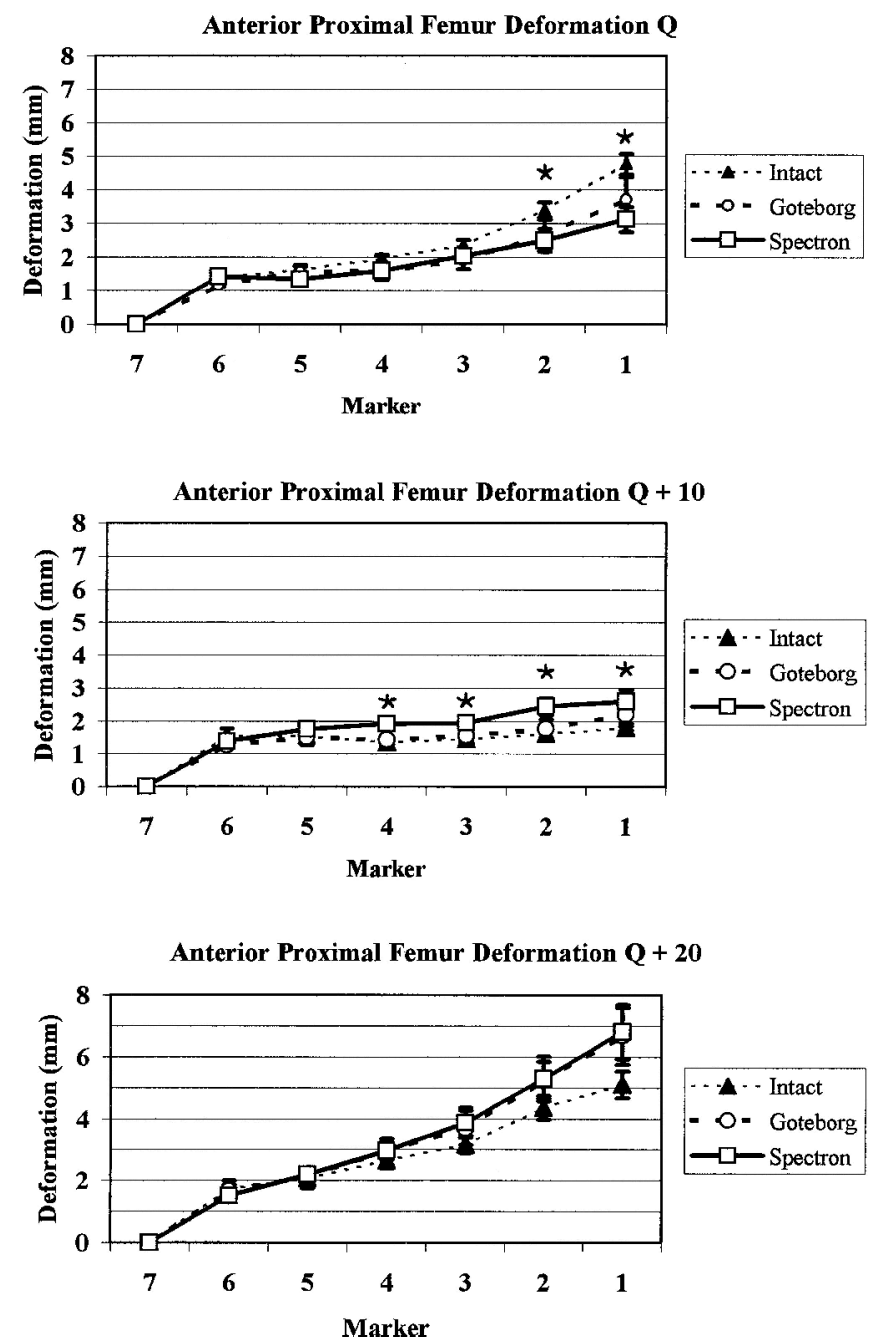

Fig. 4 Antero-posterior measures of proximal femoral deformation patterns (means \pm one standard error). * Statistically significant difference between the intact femur and the Spectron

cemented Spectron system and the cementless Gothenburg system (Table 1 ) at $Q+0^{\circ}$ adduction angle. At $Q+10^{\circ}$, the whole femoral stiffness was much greater for all constructs, but only slightly increased by the implant of either sort (and not significantly). At $Q+20^{\circ}$ the stiffness was similar to that at $Q+0^{\circ}$, and proximal replacement slightly reduced the stiffness (although again not significantly). There was an apparent trend towards less severe changes with the Gothenburg

Table 1 Whole bone stiffnesses ( N/mm, $n=5)$ for intact and implanted femora (mean \pm standard error)

\begin{tabular}{llllll}
\hline & \multicolumn{2}{c}{ Spectron } & & \multicolumn{2}{c}{ Gothenburg } \\
\cline { 2 - 3 } \cline { 5 - 6 } & Intact & Implanted & & Intact & Implanted \\
\hline$Q$ & $254 \pm 6.6^{*}$ & $324 \pm 11.3$ & & $256 \pm 10.2^{*}$ & $318 \pm 7.0$ \\
$Q+10^{\circ}$ & $329 \pm 8.5$ & $356 \pm 21.6$ & & $321 \pm 8.9$ & $338 \pm 10.2$ \\
$Q \pm 20^{\circ}$ & $275 \pm 19.2$ & $253 \pm 30.9$ & & $268 \pm 8.6$ & $251 \pm 12.0$ \\
\hline
\end{tabular}

* Statistically significant $(p \leqslant 0.05)$ comparing intact versus implanted. implant, with less variability, but again this did not attain statistical significance.

When whole bone deformation was viewed from the medial aspect, only one measure showed any statistically significant differences: that between the intact femur deformation at point 1 and both implanted construct deformations at that point (Fig. 3, $p<0.05$ ) at $Q+0^{\circ}$. At $Q+10^{\circ}$ and $Q+20^{\circ}$, no significant differences were detected in the medial view with implantation of either system.

Anterior views of the proximal deformation showed stronger effects, with statistically significant differences at both $Q+0^{\circ}$ and $Q+10^{\circ}$ loading angles (Fig. 4). At $Q+0^{\circ}$ the deformations for the Spectron constructs at points 1 and 2 were significantly different from those of the intact femur $(p<0.05)$, but the Gothenburg construct deformations were not statistically different from the intact. At $Q+10^{\circ}$, the deformations with the Spectron implant were significantly different from the intact femur at points 1, 2, 3 and 4; the deformations were significantly different from the Gothenburg system deformations at points 2, 3 and 4 . The Gothenburg deformations were not significantly different from the intact femur. At $Q+20^{\circ}$ none of the differences (between intact and implanted or between implants) attained statistical significance ( $p=0.09$, ANOVA).

Both systems showed micromotion over the first 200 cycles of loading, which settled to approximately $20 \mu \mathrm{m}$ movement (Gothenburg hip, $21 \pm 6 \mu \mathrm{m}$; Spectron, $29 \pm 6 \mu \mathrm{m}$ ), with no significant differences between the two systems (Fig. 5). Total rotational migration of the implant with respect to the femur through 1000 cycles was $64 \pm 7 \mu \mathrm{m}$ for the cementless Gothenburg hip and $17 \pm 2 \mu \mathrm{m}$ for the cemented Spectron system. These differences were statistically significant $(p<0.01)$.

\section{DISCUSSION}

The loading situation used in this study represents only the action of the resultant joint force, and does not attempt to model muscle forces. Cristofolini and colleagues [32] found that the muscle actions at the proximal femur, particularly the glutei, could significantly affect the bone strains in the medial and lateral aspects; $\mathrm{Lu}$ and co-workers verified this finding experimentally [33]. With finite element modelling of the proximal femur, Taylor et al. [34] postulated that muscle actions about the hip serve to minimize the bending stresses in the femur. They modelled this by increasing the angle of application of the joint resultant force from 13 to $20^{\circ}$, and the calculations were validated using radiographic whole-bone deformation measures. With their alignment of the femoral shaft vertically, a $13^{\circ}$ loading corresponds roughly to $Q+3^{\circ}$ in the present study, and their $20^{\circ}$ loading to the present $Q+10^{\circ}$. This implies that loading at $Q+10^{\circ}$ minimizes the bending stresses in the femur, 


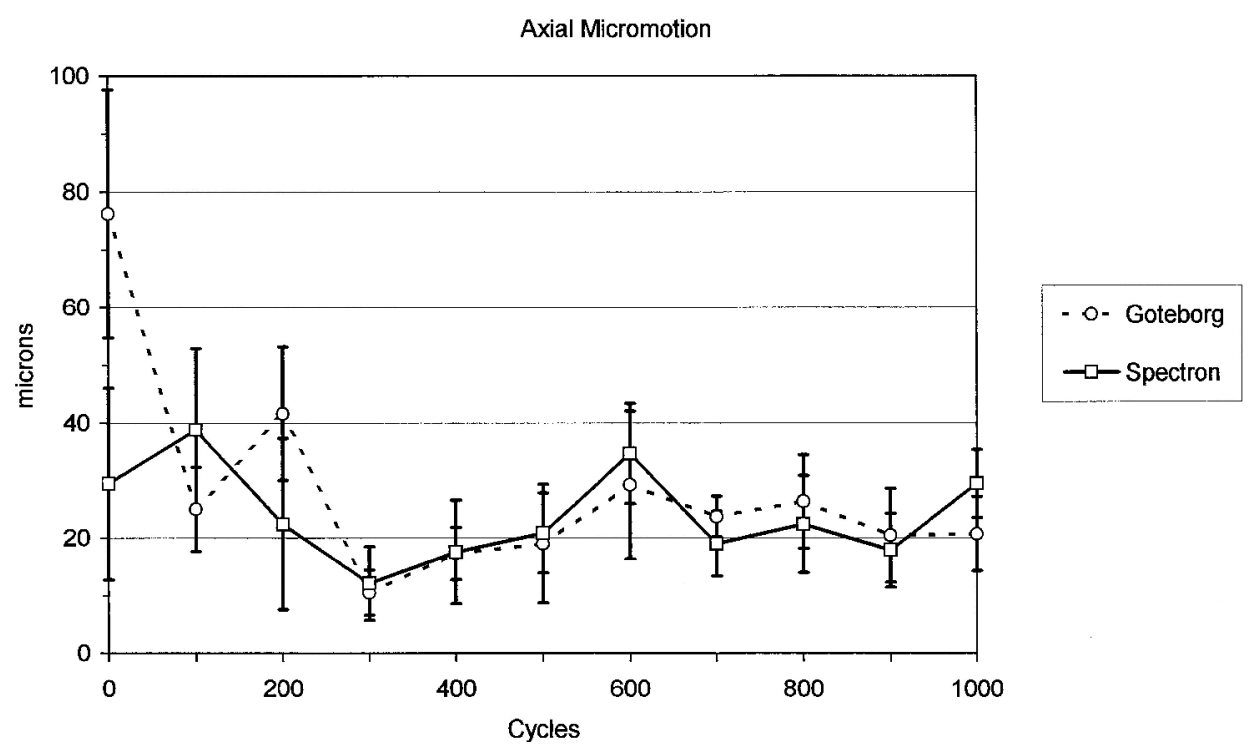

Fig. 5 Vertical micromotion measures

in a similar fashion to the in vivo situation with muscle forces added, and suggests that this is the most realistic loading.

In the present study, at the loading angle of $Q+0$ the joint reaction is acting approximately as it would during the stance phase of gait, but without any muscle actions at the proximal femur. The Spectron constructs are stiffer in this mode than the intact femora; the Gothenburg constructs were not statistically different from the intact bones. Stiffer construct behaviour shows that the deformations are reduced due to the metal stem bearing more stress for less deformation than the unoperated bone; the remaining bone carries less stress and strain, and suffers stress shielding. Where the bone deformation is not significantly different from the intact deformation, it is reasonable to assume that neither are the bone strains locally. This suggests that the proximal implant induces less stress shielding of the femur overall under this loading. It is also apparent that these differences are mainly restricted to the proximal two markers, which display the deformation of the femoral neck.

When the loading is changed to $Q+10^{\circ}$, a more realistic alignment, even greater differences are observed between the stiffer cemented construct and both the Gothenburg and intact femora; over not only the femoral neck, but also the proximal third of the femur (points 1 to 4, Fig. 4). Again the Gothenburg bone deformations were not significantly different from those of the intact bone. This again suggests that the strains induced in the bone by the Gothenburg concept more closely resemble those of the intact bone than those of the cemented construct: the reduction in the bone strains and deformation is less with this implant than with the cemented stem.

When the constructs are loaded at $Q+20^{\circ}$, the loading has now passed beyond normal ranges of adduction, and the resultant force apparently passes the mechanical axis of the femoral neck so that the bending induced in the femur is directed laterally instead of medially. In this mode of loading, both in the present study and also in the previous cementless stem study [25], the deformation of the intact and replaced femora increases quite dramatically, although all replaced constructs deform more than the intact specimens. In the present study, there is no apparent difference in the deformations as viewed medially, suggesting that the differences in deformation are more medio-laterally directed.

Whole bone deformation is a three-dimensional summation of all the individual bone element strains; these are in turn dependent on the applied loads and the whole bone anatomy. Techniques for the direct measurement of actual bone strain have been criticized for being susceptible to local anomalies such as implant contact zones [35] or regions of high strain gradient [36], especially when gauges (often $10 \mathrm{~mm}$ in size or less) are placed in only three regions [23] or fewer [37]. By contrast, whole bone deformation depends on the global strain field and is an integral over all the bone element strains accounting for their geometrical location. If loading conditions and bone geometry are reproduced (as in retesting the same bones under identical loads), the local bone strains will be in the same proportion as the overall deformation, with some localized variation if there is cortical thinning or bone weakness due to the surgery.

The medial view of the deformation pattern ignores deformations out of the sagittal plane. Since the loading situation is coaxial to the femoral axis, no bending actions anteriorly or posteriorly can be expected, so the medial view detects only the vertical component of the deformation while the A-P view represents both the vertical component and the horizontal (medial) component due to bending in the coronal plane. Thus the 
medial deformations are always less than those viewed antero-posteriorly, and the major differences between implant systems and intact bones are more clearly revealed on A-P views.

The vertical micromotions measured (Gothenburg, $21 \mu \mathrm{m}$; Spectron, $29 \mu \mathrm{m}$ ) are slightly higher than those reported in an earlier study (Infinity, $16 \mu \mathrm{m}$; Omnifit, $9 \mu \mathrm{m})$ [25], but within the range reported previously. Burke and colleagues reported vertical motions up to 30 (cementless stems) and $42 \mu \mathrm{m}$ (cemented stems) under vertical loading of $445 \mathrm{~N}$, but adding torsional loading increased these to 76 (cemented) and $280 \mu \mathrm{m}$ (cementless) [26]. Walker et al. [24] reported micromotions of $10-50 \mu \mathrm{m}$, the smallest movements for cemented stems. Similar results were reported by Schneider et al. [38], whose cemented implants generally moved less than cementless devices $(0.4 \mathrm{~mm}$ versus $0.4-0.8 \mathrm{~mm})$ especially in subsidence, where movements of up to $3 \mathrm{~mm}$ were measured with cementless stems. The axial micromotion measured here for the Gothenburg system was not significantly different from the cemented or cementless devices (Spectron, Omnifit or Infinity [25]), and was well within the tolerable limits of bone ingrowth response (40 [26]-150 $\mu \mathrm{m}[\mathbf{3 9}, \mathbf{4 0}]$ ). It is therefore concluded that these results indicate adequate initial stability for optimal bone response in normal clinical bone.

Verification of the improved bone strains with such a proximal stem design is being undertaken with computer tomography scanning of femora and finite element modelling of the scanned bones, and strain gauge studies of femora. These will be reported separately.

\section{CONCLUSIONS}

1. Femoral replacement with an osseointegrated implant minimizes the changes caused in the proximal femoral deformation pattern, reducing stress shielding and subsequent bone resorption.

2. Micromotions measured in both systems are less than the maximum tolerable for adequate bone ingrowth or ongrowth.

\section{ACKNOWLEDGEMENTS}

The authors would like to acknowledge with gratitude the cheerful assistance and technical contribution of Matt Sandusky, Bruce Yang and Ali Utkan during the laboratory tests in Long Beach, Stig Wennberg's continued technical contribution with design and preparation of implants and instruments, and the excellent graphics of Yutaka Kataoka.

\section{REFERENCES}

1 Bocco, F., Langan, P. and Charnley, J. Changes in the calcar femoris in relation to cement technology in total hip replacement. Clin. Orthop., 1977, 128, 287-295.

2 Engh, C. A. and Bobyn, D. J. The influence of stem size and extent of porous coating on femoral bone resorption after primary cementless hip arthroplasty. Clin. Orthop., 1988, 231, 7-28.

3 Jones, L. C. and Hungerford, D. S. Cement disease. Clin. Orthop., 1987, 225, 192-206.

4 Hungerford, D. S. and Jones, L. C. The rationale of cementless revision of cemented arthroplasty failures. Clin. Orthop., 1988, 235, 12-24.

5 Schmalzried, T. P., Jasty, M. and Harris, W. H. Periprosthetic bone loss in total hip arthroplasty: polyethylene wear debris and the concept of the effective joint space. J. Bone Jt Surg. (Am.), 1992, 74-A, 849-863.

6 Diegel, P. D., Daniels, A. U. and Dunn, H. K. Initial effect of collarless stem stiffness on femoral bone strain. J. Arthroplasty, 1989, 4(2), 173-178.

7 Engh, C. A. et al. Quantifiction of implant micromotion, strain shielding, and bone resorption with porous-coated anatomic medullary locking femoral prostheses. Clin. Orthop., 1992, 231, 13-29.

8 Huiskes, R., Weinans, H. and Dalstra, M. Adaptive bone remodelling and biomechanical design considerations for noncemented total hip arthroplasty. Orthopedics, 1989, 12, $1255-1267$.

9 Cohen, B. and Rushton, N. Bone remodelling in the proximal femur after Charnley total hip arthroplasty. $J$. Bone $J t$ Surg. (Br.), 1995, 77-B(5), 815-819.

10 Kobayashi, S., Eftekhar, N. S. and Terayama, K. Long-term bone remodelling around the Charnley femoral prostheses. Clin. Orthop., 1996, 326, 162-173.

11 Lewis, J. L. et al. The influence of prosthetic stem stiffness and of a calcar collar on stresses in the proximal end of the femur with a cemented femoral component. $J$. Bone $J t$ Surg. (Am.), 1984, 66-A, 280-286.

12 Vail, T. P. et al. The effect of hip stem material modulus on surface strain in human femora. J. Biomechanics, 1988, 31, 619-628.

13 Markolf, K. L., Amstutz, H. C. and Hirschowitz, D. L. The effect of calcar contact on femoral component micromovement. J. Bone Jt Surg. (Am.), 1980. 62-A, 1315-1323.

14 Musgrave, D. S. et al. Effects of coronally slotted femoral prostheses on cortical bone strain. J. Arthroplasty, 1997, 12(6), 657-669.

15 Smith, E. and Harris, W. H. Increasing prevalence of femoral lysis in cementless total hip arthroplasty. J. Arthroplasty, 1995, 10(4), 407-412.

16 Maloney, W. J. and Woolson, S. T. Increasing incidence of femoral osteolysis in association with uncemented HarrisGalante total hip arthroplasty. J. Arthroplasty, 1996, 11(2), 130-134.

17 Albrektsson, T. et al. Osseointegrated titanium implants. Acta Orthopaedica Scand., 1981, 52, 155-170.

18 Albrektsson, T. and Jacobsson, M. Bone metal interface in osseointegration. J. Prosthetic Dentistry, 1987, 57(5), 597-607.

19 Albrektsson, T. and Albrektsson, B. Osseointegration of 
bone implants. Acta Orthopaedica Scand., 1987, 58, 567-577.

20 Carlsson, L. V. et al. Osseointegration of titanium implants. Acta Orthopaedica Scand., 1986, 57, 285-289.

21 Carlsson, L. V. et al. Removal torques for polished and rough titanium implants. Int. J. Oral Maxillofacial Implants, 1988, 3(1), 21-24.

22 Röstlund, T. et al. Osseointegrated knee prostheses. Scand. J. Plastic Reconstructive Surg., 1989, 23, 43-46.

$23 \mathrm{Oh}$, I. and Harris, W. H. Proximal strain distribution in the loaded femur. J. Bone Jt Surg. (Am.), 1978, 60-A(1), 75-85.

24 Walker, P. S. et al. Strains and micromotions of press-fit femoral stem prostheses. J. Biomechanics, 1987, 20(7), 693-702.

25 Lee, T. Q. et al. Structural integrity of implanted WMT Infinity hip and Osteonics Omnifit hip in fresh cadaveric hips. J. Biomed. Mater. Res., 1998, 39, 516-523.

26 Burke, D. W. et al. Micromotion of cemented and uncemented femoral components. J. Bone Jt Surg. (Br.), 1991, 73-B, 33-37.

27 Phillips, T. W., Messieh, S. S. and McDonald, P. D. Femoral stem fixation in hip replacement. J. Bone Jt Surg. (Br.), 1990, 72-B(3), 431-434.

28 Malchau, H., Herberts, P. and Ahnfelt, L. Prognosis of total hip replacement in Sweden. Acta Orthopaedica Scand., 1993, 64(5), 497-506.

29 Albrektsson, T. et al. Gothenburg osseointegrated hip arthroplasty. Clin. Orthop., 1998, 352, 81-94.

30 Lee, T. Q. et al. Assessment of geometry and deformation characteristics of human cadaveric femur. Adv. Bioengng, ASME, 1992, 74, 839-848.
31 Davy, D. T. et al. Telemetric force measurements across the hip after total arthroplasty. J. Bone Jt Surg. (Am.), 1988, 70-A, 45-50.

32 Cristofolini, L. et al. Influence of thigh muscles on the axial strains in a proximal femur during early stance in gait. J. Biomechanics, 1995, 28(5), 617-624.

33 Lu, T.-W. et al. Influence of muscle activity on the forces in the femur: an in vivo study. J. Biomechanics, 1997, 30, 1101-1106.

34 Taylor, M. E. et al. Stress and strain distribution within the intact femur: compression or bending? Med. Engng Physics, 1996, 18(2), 122-131.

35 Zhou, X. M., Walker, P. S. and Robertson, D. D. Effect of press-fit femoral stems on strains in the femur. J. Arthroplasty, 1990, 5(1), 71-82.

36 Finlay, J. B. et al. An evaluation of three loading configurations for the in vitro testing of femoral strains in total hip arthroplasty. J. Orthop. Res., 1991, 9(5), 749-759.

37 Szivek, J. A. and Gealer, R. L. Technical note: comparison of the deformation response of synthetic and cadaveric femora during simulated one-legged stance. J. Appl. Biomater., 1991, 2, 277-280.

38 Schneider, E. et al. A comparative study of the initial stability of cementless hip prostheses. Clin. Orthop., 1989, 248, 200-209.

39 Callaghan, J. J. et al. The effect of femoral stem gemoetry on interface motion in uncemented porous-coated total hip prostheses. J. Bone Jt Surg. (Am. ), 1992, 74-A(6), 839-848.

40 Pilliar, R. M., Lee, J. M. and Maniatopoulos, C. Observations on the effect of movement on bone ingrowth into porous-surfaced implants. Clin. Orthop., 1986, 208, $108-113$. 